OPEN ACCESS

Edited by:

Rayaz A. Malik,

Weill Cornell Medicine-Qatar, Qatar

Reviewed by:

Georgios Ponirakis,

Weill Cornell Medicine-Qatar, Qatar

Ashfaq Shuaib,

University of Alberta, Canada

${ }^{*}$ Correspondence:

Xin $\mathrm{Li}$

lixin_dhuf@163.com

Specialty section: This article was submitted to Clinical Diabetes,

a section of the journal

Frontiers in Endocrinology

Received: 18 August 2020 Accepted: 18 November 2020 Published: 21 December 2020

Citation:

Wang $D-Q$, Wang $L$, Wei $M-M$, Xia X-S, Tian X-L, Cui X-H and LiX (2020) Relationship Between Type 2 Diabetes and White Matter Hyperintensity: A Systematic Review.

Front. Endocrinol. 11:595962. doi: 10.3389/fendo.2020.595962

\section{Relationship Between Type 2 Diabetes and White Matter Hyperintensity: A Systematic Review}

\author{
Dan-Qiong Wang ${ }^{1,2}$, Lei Wang ${ }^{1}$, Miao-Miao Wei ${ }^{2}$, Xiao-Shuang $\mathrm{Xia}^{2}$, Xiao-Lin Tian ${ }^{2}$, \\ Xiao-Hong Cui ${ }^{3}$ and ${\mathrm{Xin} \mathrm{Li}^{2 *}}^{\text {* }}$ \\ ${ }^{1}$ Department of General Medical, Shanxi Bethune Hospital Shanxi Academy of Medical Sciences, Taiyuan, China, \\ ${ }^{2}$ Department of Neurology, The Second Hospital of Tianjin Medical University, Tianjin, China, ${ }^{3}$ Department of Psychiatry, \\ Shanxi Bethune Hospital Shanxi Academy of Medical Sciences, Taiyuan, China
}

White matter (WM) disease is recognized as an important cause of cognitive decline and dementia. White matter lesions (WMLs) appear as white matter hyperintensities (WMH) on T2-weighted magnetic resonance imaging (MRI) scans of the brain. Previous studies have shown that type 2 diabetes (T2DM) is associated with $\mathrm{WMH}$. In this review, we reviewed the literature on the relationship between T2DM and WMH in PubMed and Cochrane over the past five years and explored the possible links among the presence of T2DM, the course or complications of diabetes, and WMH. We found that: (1) Both from a macroand micro-scopic point of view, most studies support the relationship of a larger WMH and a decrease in the integrity of $\mathrm{WMH}$ in T2DM; (2) From the relationship between brain structural changes and cognition in T2DM, the poor performance in memory, attention, and executive function tests associated with abnormal brain structure is consistent; (3) Diabetic microangiopathy or peripheral neuropathy may be associated with $\mathrm{WMH}$, suggesting that the brain may be a target organ for T2DM microangiopathy; (4) Laboratory markers such as insulin resistance and fasting insulin levels were significantly associated with $\mathrm{WMH}$. High $\mathrm{HbA} 1 \mathrm{c}$ and high glucose variability were associated with $\mathrm{WMH}$ but not glycemic control.

\section{Keywords: type 2 diabetes mellitus, white matter hyperintensities, relationship, microscopic, cognitive function,} microangiopathy and neuropathy

\section{INTRODUCTION}

In 1987, Hachinski et al. first used the low density of computed tomography to represent diffuse white matter changes, called leukoaraiosis (LA) or rare white matter (1). White matter hyperintensities (WMH) are defined as patchy areas of signal hyperintensity scattered in the deep or periventricular white matter evident on brain magnetic resonance imaging (MRI) T2weighted or Fluid Attenuation Inversion Recovery (FLAIR) sequences (2). This high signal on MRI is caused by cerebral small vessel disease (CSVD), also known as white matter lesions (WML) (3-5). Therefore, different terms such as WMH, WML, white matter disease, and LA are considered synonyms (2). We use the term WMH in this review. 
$\mathrm{WMH}$ prevalence was shown to increase with age (6). The prevalence of $\mathrm{WMH}$ is as high as $70 \%$ in Chinese population aged between 35 and 80 years (7), and nearly 90\% in the European population aged between 60 and 90 years (8). In a meta-analysis study, WMH were reported to be linked to an increased risk of stroke, dementia, and death (9). Therefore, understanding the causes of WMH is essential for the prevention of cerebrovascular disease. Advanced age and hypertension are closely associated with WMH burden (10-12). The relationship between diabetes and the $\mathrm{WMH}$ burden remains controversial.

Type 2 diabetes mellitus (T2DM) prevalence has been increasing rapidly over the past few decades worldwide (13).Based on recent International Diabetes Federation (IDF) estimates, in 2017, there were 451 million adults (age: 18-99 years) with diabetes globally, a figure that is predicted to reach 693 million by $2045(14,15)$. T2DM is considered a risk factor for cerebrovascular disease (16). There is strong evidence that T2DM is associated with an increased risk of stroke $(17,18)$, dementia (19), depression, and cognitive impairment $(20,21)$. It is also thought to be related to CSVD, such as WMHs and brain atrophy (22-25).

In this review, we assessed the relationship between T2DM and $\mathrm{WMH}$, and the impact of decreased white matter integrity on cognition in patients with T2DM. Del Bene et al. (26) investigated the correlation between T2DM and LA in 2015 and found that the relationship between LA and T2DM was more consistent than previously reported. However, due to the heterogeneity of the research methods, no clear conclusions were reached. Therefore, we searched the recent relevant literature and conducted a systematic review and comprehensive analysis again.

\section{METHODS}

We use the following term combinations: white matter lesion MRI diabet $^{*}$, white matter hyperintensity white matter hyperintensities MRI diabet*, white matter change OR white matter changes AND MRI AND diabet*, and leukoaraiosis AND MRI AND diabet* ${ }^{*}$ A literature search was conducted using PubMed and Cochrane.

Inclusion criteria: (1) Recently published English literature; (2) Definite imaging findings of T2DM or prediabetes and abnormal white matter structure; (3) White matter structural abnormalities measured by MRI; (4) Quantitative measurements of the association between T2DM or prediabetes and abnormal white matter structure were provided; (5) Cross-sectional, casecontrol or cohort epidemiological studies or Mendelian randomization studies were used.

Exclusion criteria: (1) Articles not focusing on the relationship between T2DM and white matter lesions; (2) The same literature retrieved by different search terms; (3) Articles on the relationship between type 1 diabetes mellitus and white matter lesions; (4) Duplicate publications or review articles of the same data set; (5) Articles dealing with animal research and genetic factors; (6) Review articles.

Quality assessment: The methodological quality of the crosssectional studies included was assessed using an 11-item checklist that was recommended by the Agency for Healthcare Research and Quality (AHRQ). An item would be scored " 0 " if it was answered "NO" or "UNCLEAR"; if it was answered "YES", then the item scored " 1 ". Article quality was assessed as follows: low quality $=0-3$; moderate quality $=4-7$; high quality $=8-11$. The Newcastle-Ottawa Scale (NOS) was used to assess each of the cohort studies' quality by two independent authors. The NOS consists of three parts: selection (0-4 points), comparability ( $0-2$ points), and outcome assessment ( $0-3$ points). NOS scores of six were assigned as high-quality studies.

We explored the possible links between the presence of T2DM, the duration or complications of diabetes, laboratory markers such as glycated hemoglobin concentration, insulin resistance, and WMH or its progression over time. In addition, we also considered the effect of the relationship between T2DM and $\mathrm{WMH}$ on cognitive function.

\section{RESULTS}

\section{Literature Information}

A literature search was conducted using PubMed and Cochrane, and 478 articles published between January 2015 and December 2019 were retrieved. After title and abstract screening, 117 articles were retrieved, and 38 articles were finally included, including two obtained from the retrieved literature references (Figure 1).

In the included studies, WMH is evaluated by conventional MRI sequences. The volume was measured by visual rating scales, automatic or semi-automatic segmentation methods, or through unconventional MRI modalities, such as MRI-DTI or diffusion kurtosis imaging (DKI) methods. In each of the included studies, we recorded the study design, sample size, mean age, neuroimaging methods, study results, and correction parameters (Table 1).

\section{Bias Risk Results for All Studies Included}

All cross-sectional studies were assessed by AHRQ, eight of which were of high quality, and 30 of which were of moderate quality. All cohort studies were assessed by NOS, three of which were of high quality, and the rest were of medium quality.

\section{Macroscopic Analysis of the Relationship Between Type 2 Diabetes Mellitus and White Matter Hyperintensities}

We define macro-analysis as the analysis of WMH volume in patients with T2DM by conventional MRI sequences (T1, T2, and FLAIR). Macroscopically, most studies have observed a larger WMH in T2DM. A recent cross-sectional studies (32, $40,42,55)$ using conventional MRI sequences and double inversion recovery (DIR) sequences have shown that patients with diabetes had more WMHs than the control group. In a prospective cohort study based on the elderly population (24), Marseglia et al. found that diabetes was cross-sectionally associated with larger WMH volume. Longitudinally, diabetes was associated with faster WMH accumulation. These findings 


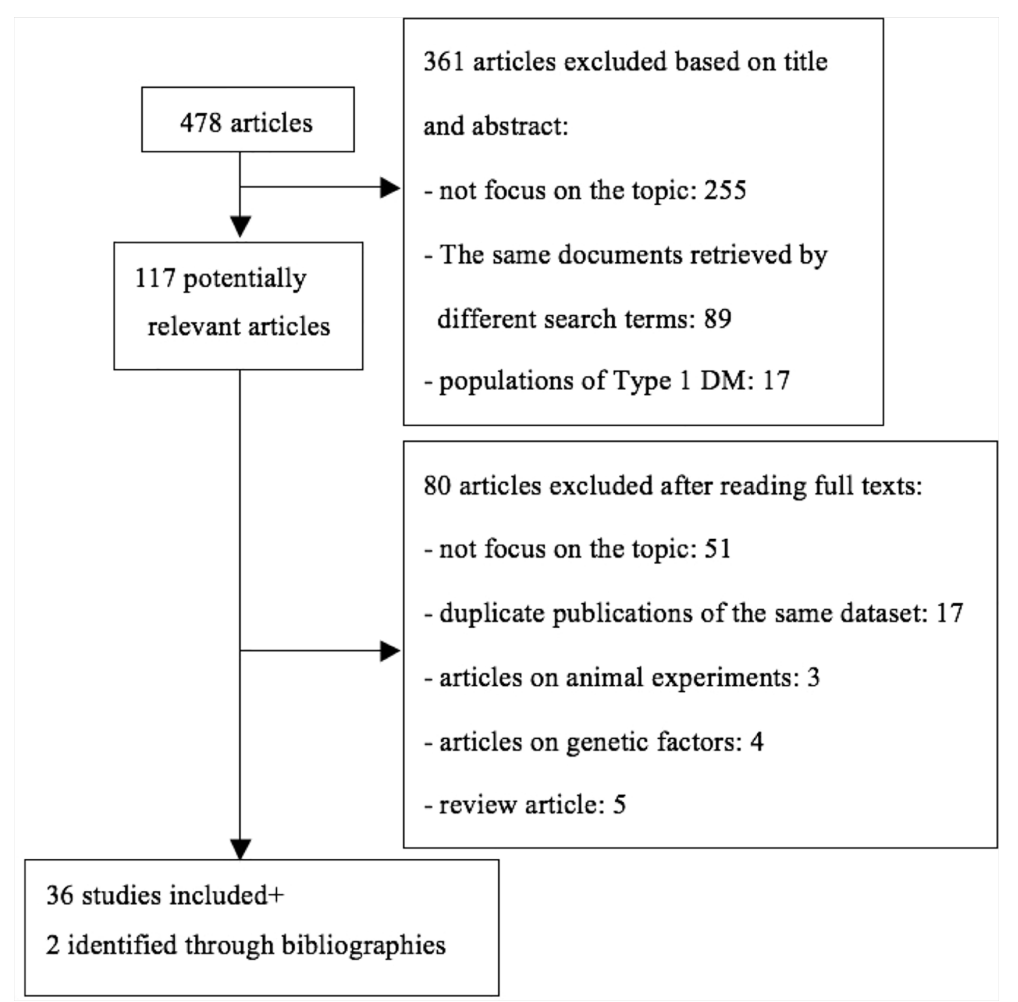

FIGURE 1 | Summary of literature search.

highlight that diabetes may increase the accumulation of $\mathrm{WMH}$ markedly over time. Another middle-aged cohort study (38) showed that prediabetes and T2DM were significantly associated with larger volumes of WMHs compared with normal glucose metabolism (NGM). These results indicate that in middle-aged populations, structural brain abnormalities already occur in prediabetes. In a large community cross-sectional study (52), Schneider et al. found that ARIC-NCS participants with diabetes with $\mathrm{HbAlc} \geq 7.0 \%$ have an increased burden of $\mathrm{WMH}$, but those with prediabetes (HbA1c 5.7 to $<6.5 \%$ ) and diabetes with HbAlc $<7.0 \%$ have WMH similar to those without diabetes.

Most studies on WMH have examined WMH volume, sometimes also addressing the number, location, and shape of WMH. Pierleone Lucatelli et al. (28) calculated WML volume and the number of lesions on FLAIR images using a semiautomated segmentation technique, and demonstrating a statistically significant correlation between T2DM and WMH in terms of lesion number and volume. Jeroen de Bresser et al. (27) artificially segmented the WMH of FLAIR images. With the marching cubes algorithm, a mesh is created to determine $\mathrm{WMH}$ shape and location features. In this study, T2DM had more nonpunctuate $\mathrm{WMH}$ and a difference in shape (eccentricity) of punctuate deep WMH compared to controls, but patients with T2DM did not differ from controls on traditional $\mathrm{WMH}$ measures (total WMH volume).

However, it may be affected by the selection bias of imaging analysis methods and study populations. There are also studies showing no convincing link between T2DM and higher WMH. A multiethnic cohort of individuals surveyed (43) showed that T2DM was not convincingly associated with a higher lesion load, either when considering the population as a whole or in SouthAsian or European subgroups separately. Sundar U's study (49) also showed that among both diabetics and non-diabetics, no difference in periventricular white matter hyperintensities was found. Deep white matter hyperintensities were, conversely, seen only in the control group. Data from a prospective cohort study (25) also showed that a history of diabetes was associated with decreased white matter hyperintensity volume.

\section{Microscopic Analysis of the Relationship Between Type 2 Diabetes Mellitus and White Matter Hyperintensities}

At present, diffusion tensor imaging (DTI) technology is mainly used to measure the microstructural changes of WM. We define micro-analysis as observed a decreased in the integrity of WMH in T2DM by DTI or DKI technology. The measures most often analyzed by DTI are fractional anisotropy (FA) and mean diffusivity (MD). Fractional anisotropy (FA) reflects the restriction of water molecule movement in all directions. Mean diffusivity $(\mathrm{MD})$ is a scalar measure of how quickly water molecules diffuse overall $(63,64)$. The lower the FA, the higher the MD and the worse the microstructural integrity of WM. The Rotterdam Study (29) is a prospective cohort study based on the middle-aged and elderly population, which found significant 
TABLE 1 | The study design, sample size, mean age, neuroimaging methods, study results, and correction parameters in the included studies.

\begin{tabular}{|c|c|c|c|c|c|c|c|c|c|}
\hline Number & Study & $\begin{array}{l}\text { Study } \\
\text { Design }\end{array}$ & $\begin{array}{c}\text { Subjects }(\mathrm{n}) \\
\text { total/type } 2 \\
\text { DM }\end{array}$ & $\begin{array}{l}\text { Mean age } \\
\text { (years) }\end{array}$ & Imaging & $\begin{array}{c}\text { WMH } \\
\text { assessment } \\
\text { method }\end{array}$ & Results & P-value & Adjustments \\
\hline 1 & $\begin{array}{l}\text { de Bresser } \\
\text { et al. (27) }\end{array}$ & CS & $114 / 60$ & 71 & MRI-FLAIR & WMH shape & $\begin{array}{l}\text { a different shape } \\
\text { (eccentricity) of } \\
\text { punctuate deep } \\
\text { WMH in T2DM }\end{array}$ & $\begin{array}{l}\text { (Beta }(95 \% \mathrm{Cl}) \text { : } \\
0.40 \\
(0.23 \leftrightarrow 0.58))\end{array}$ & No \\
\hline 2 & $\begin{array}{l}\text { Lucatellia } \\
\text { et al. (28) }\end{array}$ & CS & $93 / 19$ & $71 \pm 9$ & MRI-FLAIR & $\begin{array}{l}\text { WML volume } \\
\text { and number }\end{array}$ & $\begin{array}{l}\text { WMLV } \\
\text { associated with } \\
\text { diabetes } \\
\text { WMLN } \\
\text { associated with } \\
\text { diabetes }\end{array}$ & $\begin{array}{l}P<0.0001 \\
P=0.001\end{array}$ & $\begin{array}{l}\text { age, smoking status, gender, } \\
\text { hypertension, hyperlipidemia, } \\
\text { Coronary artery disease, } \\
\text { Ischemic event }\end{array}$ \\
\hline 3 & $\begin{array}{l}\text { de Groot } \\
\text { et al. (29) }\end{array}$ & CS & $4532 / 406$ & 63.8 & MRI-DTI & FA & $\begin{array}{l}\text { FA reduction } \\
\text { (the association } \\
\text { tracts and the } \\
\text { forceps minor } \\
\text { WM)in DM }\end{array}$ & $\begin{array}{l}\text { Bonferroni } \\
\text { correction }\end{array}$ & age,WMH \\
\hline 4 & $\begin{array}{l}\text { Ferik et al. } \\
\text { (30) }\end{array}$ & $L$ & $136 / 66$ & $54.5 \pm 7.6$ & $\begin{array}{l}\text { MRI-T1T2 } \\
\text { FLAIR }\end{array}$ & DWMLs & $\begin{array}{l}\text { DWMLs were } \\
\text { more frequent in } \\
\text { patients with } \\
\text { polyneuropathy }\end{array}$ & $p=0.016$ & age, duration of DM \\
\hline 5 & $\begin{array}{l}\text { Groeneveld } \\
\text { et al. (31) }\end{array}$ & CS & $\begin{array}{c}\text { 48(T2DM)/ } \\
\text { 25(T2DM } \\
\text { with } \mathrm{MCl} \text { or } \\
\text { early } \\
\text { dementia }\end{array}$ & $\begin{array}{c}76.4 \pm 5.0 \\
\text { (T2DM with } \\
\text { cognitive } \\
\text { impairment) } \\
76.5 \pm 4.8 \\
\text { (without } \\
\text { cognitive } \\
\text { impairment) }\end{array}$ & MRI-DTI & $\begin{array}{l}\text { WMH volume } \\
\text { FA MD }\end{array}$ & No association & NS & $\mathrm{NO}$ \\
\hline 6 & $\begin{array}{l}\text { Marseglia } \\
\text { et al. (24) }\end{array}$ & $\begin{array}{l}\text { CS } \\
L\end{array}$ & $\begin{array}{l}\text { 2746/1189 } \\
\text { (Prediabetes } \\
\text { and } \\
\text { diabetes) }\end{array}$ & $\begin{array}{c}74.1 \pm 9.5 \\
\text { (Diabetes) } \\
74.6 \pm 10.4 \\
\text { (Prediabetes) } \\
71.3 \pm 10.0 \\
\text { (Diabetesfree) }\end{array}$ & MRI-FLAIR & WMHV & $\begin{array}{l}\text { DM associated } \\
\text { with larger } \\
\text { WMHV } \\
\text { diabetes was } \\
\text { associated with } \\
\text { a faster increase } \\
\text { in WMHV }\end{array}$ & $\begin{array}{l}p=0.004 \\
p=0.023\end{array}$ & $\begin{array}{l}\text { sex, education, SES, BMI, } \\
\text { hypertension, } \\
\text { heart disease }\end{array}$ \\
\hline 7 & $\begin{array}{l}\text { Raffield } \\
\text { et al. (32) }\end{array}$ & CS & $784 / 682$ & 65.89 & MRI-T1 DTI & $\begin{array}{l}\text { WMLV、 } \\
\text { FA、MD }\end{array}$ & $\begin{array}{l}\text { increased } \\
\text { WMLV inT2DM } \\
\text { reduced white } \\
\text { matter FA } \\
\text { inT2DM } \\
\text { increased white } \\
\text { matter MD } \\
\text { inT2DM }\end{array}$ & $\begin{array}{l}p=0.008 \\
p \leq 0.001 \\
p \leq 0.031\end{array}$ & $\begin{array}{l}\text { age, scanner, sex, history of } \\
\text { cardiovascular disease, } \\
\text { smoking, statin use, educational } \\
\text { attainment, and blood pressure } \\
\text { medication use }\end{array}$ \\
\hline 8 & $\begin{array}{l}\text { Freedman } \\
\text { et al. (33) }\end{array}$ & CS & $584 / 584$ & $60.1 \pm 7.9$ & $\begin{array}{l}\text { MRI- } \\
\text { T1T2FLAIR }\end{array}$ & WMLV & $\begin{array}{l}\text { higher UACR } \\
\text { with higher } \\
\text { WMLV } \\
\text { higher eGFR } \\
\text { with lower } \\
\text { WMLV }\end{array}$ & $\begin{array}{l}P=0.05 \\
P<0.001\end{array}$ & $\begin{array}{l}\text { age, sex, level of education, } \\
\text { body mass index, hemoglobin } \\
\text { A1c concentration, hypertension, } \\
\text { diabetes duration, smoking } \\
\text { status, history of cardiovascular } \\
\text { disease, eGFR, UACR }\end{array}$ \\
\hline 9.1 & $\begin{array}{l}\text { Hughes } \\
\text { et al. (34) }\end{array}$ & CS & $584 / 584$ & $60.1 \pm 7.9$ & $\begin{array}{l}\text { MRI- } \\
\text { T1T2FLAIR }\end{array}$ & WMLV & $\begin{array}{l}\text { Higher WMV } \\
\text { with } \\
\text { poorer3MSE/ } \\
\text { MMSE } \\
\text { performance } \\
\text { higher Stroop } \\
\text { interference }\end{array}$ & $\begin{array}{l}p=3.6 \times 10^{-3} \\
p=7.5 \times 10^{-3}\end{array}$ & $\begin{array}{l}\text { age, sex, BMI, hemoglobin A1c, } \\
\text { hypertension, smoking, CVD, } \\
\text { diabetes duration, LDL- } \\
\text { cholesterol of education, MRI } \\
\text { scanner, total intracranial volume }\end{array}$ \\
\hline 9.2 & $\begin{array}{l}\text { Whitlow } \\
\text { et al. (35) }\end{array}$ & CS & $263 / 263$ & 60.4 & $\begin{array}{l}\text { MRI- } \\
\text { T1FLAIR }\end{array}$ & WMLV & $\begin{array}{l}\text { Higher WMLV } \\
\text { was associated } \\
\text { with poorer } \\
\text { performance }\end{array}$ & $P<0.05$ & $\begin{array}{l}\text { age, sex, body mass index, } \\
\text { HbA1c, level of education }\end{array}$ \\
\hline 10 & $\begin{array}{l}\text { Liang et al. } \\
\text { (36) }\end{array}$ & CS & $\begin{array}{c}60 / 30 \\
\text { (Prediabetes) }\end{array}$ & $\begin{array}{l}55.03 \pm 6.66 \\
\text { (Prediabetes)/ }\end{array}$ & MRI-DTI & FA & $\begin{array}{l}\text { the } F A(b C C \\
\text { SLF.R SLF.L) }\end{array}$ & $\begin{array}{l}p=0.035 p=0.047 \\
p=0.040\end{array}$ & NO \\
\hline
\end{tabular}


TABLE 1 | Continued

\begin{tabular}{|c|c|c|c|c|c|c|c|c|c|}
\hline Number & Study & $\begin{array}{l}\text { Study } \\
\text { Design }\end{array}$ & $\begin{array}{c}\text { Subjects (n) } \\
\text { total/type } 2 \\
\text { DM }\end{array}$ & $\begin{array}{l}\text { Mean age } \\
\text { (years) }\end{array}$ & Imaging & $\begin{array}{c}\text { WMH } \\
\text { assessment } \\
\text { method }\end{array}$ & Results & $\mathrm{P}$-value & Adjustments \\
\hline & & & & $\begin{array}{l}52.80 \pm 7.53 \\
\quad(\text { Control })\end{array}$ & & & $\begin{array}{l}\text { decreased in } \\
\text { the prediabetic } \\
\text { group }\end{array}$ & & \\
\hline 11 & $\begin{array}{l}\text { Vergoossen } \\
\text { et al. (37) }\end{array}$ & CS & $\begin{array}{l}\text { 2219/858 } \\
\text { (prediabetes } \\
\text { and } \\
\text { diabetes) }\end{array}$ & $59 \pm 8$ & $\begin{array}{l}\text { MRI WM } \\
\text { tractography }\end{array}$ & node degree & $\begin{array}{l}\text { lower local } \\
\text { efficiency with } \\
\text { prediabetes } \\
\text { lower clustering } \\
\text { coefficient with } \\
\text { prediabetes } \\
\text { higher } \\
\text { communicability } \\
\text { withT2DM }\end{array}$ & $\begin{array}{l}P=0.033 \\
P=0.049 \\
P=0.008\end{array}$ & $\begin{array}{l}\text { age, sex, education, average } \\
\text { node degree, MRI date }\end{array}$ \\
\hline 12 & $\begin{array}{l}\text { van Agtmaal } \\
\text { et al. (38) }\end{array}$ & CS & $\begin{array}{l}\text { 2,228/855 } \\
\text { (prediabetes } \\
\text { and } \\
\text { diabetes) }\end{array}$ & $59.2 \pm 8.2$ & $\begin{array}{l}\text { MRI-T1T2 } \\
\text { FLAIR }\end{array}$ & WMHV & $\begin{array}{l}\text { larger WMHVs } \\
\text { (dWMHs and } \\
\text { pWMHs) with } \\
\text { prediabetes and } \\
\text { T2DM }\end{array}$ & Ptrend $<0.001$ & $\begin{array}{l}\text { age, sex, intracranial volume, } \\
\text { time between baseline and MRI } \\
\text { measurements, BMl, smoking } \\
\text { status, total-to-HDL cholesterol } \\
\text { ratio, office systolic blood } \\
\text { pressure, estimated glomerular } \\
\text { filtration rate, educational level }\end{array}$ \\
\hline 13 & $\begin{array}{l}\text { Hsu et al. } \\
\text { (39) }\end{array}$ & CS & $\begin{array}{c}1,204 \\
\text { (diabetes) }\end{array}$ & $62.8 \pm 10.3$ & MRI & WMHV & $\begin{array}{l}\text { WMLV higher in } \\
\text { African } \\
\text { Americans than } \\
\text { European } \\
\text { Americans }\end{array}$ & $p=0.001$ & $\begin{array}{l}\text { age, sex, diabetes duration, BMI, } \\
\text { HbA1c, total intracranial volume, } \\
\text { scanner, statins, CVD, smoking, } \\
\text { hypertension }\end{array}$ \\
\hline 14 & $\begin{array}{l}\text { Weinstein } \\
\text { et al. ( } 40)\end{array}$ & CS & $\begin{array}{l}\text { 1,597/124 } \\
\text { (diabetes } \\
\text { andFBG) }\end{array}$ & $40.3 \pm 8.5$ & $\begin{array}{l}\text { MRI-FLAIR } \\
\text { DTI }\end{array}$ & WMHV FA & $\begin{array}{l}\text { larger WMHV } \\
\text { with diabetes } \\
\text { lower FA with } \\
\text { diabetes and } \\
\text { greater FBG }\end{array}$ & $\begin{array}{l}P=0.015 \\
P<0.05\end{array}$ & $\begin{array}{l}\text { age, sex, time between } \\
\text { examination } 1 \text { and } \mathrm{MRI}\end{array}$ \\
\hline 15 & $\begin{array}{l}\text { Ben } \\
\text { Assayag } \\
\text { et al. (41) }\end{array}$ & $L$ & $392 / 118$ & $67.4 \pm 9.7$ & MRI- DTI & FA MD & $\begin{array}{l}\text { decreased } \\
\text { NAWM FA with } \\
\text { T2DM } \\
\text { increased } \\
\text { NAWM MD with } \\
\text { T2DM }\end{array}$ & $\begin{array}{l}p=0.006 \\
p=0.003\end{array}$ & $\mathrm{NO}$ \\
\hline 16 & $\begin{array}{l}\text { Lin et al. } \\
(42)\end{array}$ & CS & 4,683/1102 & $64.3 \pm 9.5$ & MRI-FLAIR & LA & $\begin{array}{l}\text { LA early onset } \\
\text { with diabetes } \\
\text { LA late } \\
\text { progression with } \\
\text { diabetes }\end{array}$ & $\begin{array}{l}P<0.001 \\
P<0.05\end{array}$ & NO \\
\hline 17 & $\begin{array}{l}\text { Sanahuja } \\
\text { et al. (43) }\end{array}$ & CS & 312(153DR) & $\begin{array}{l}57 \text { (Diabetes } \\
\text { without DR) } \\
61 \text { (Diabetes } \\
\text { with DR) }\end{array}$ & $\begin{array}{l}\text { MRI-T2 } \\
\text { FLAIR }\end{array}$ & WMLs & $\begin{array}{l}\text { higher WML in } \\
\text { patients with DR }\end{array}$ & $p=0.04$ & NO \\
\hline 18 & $\begin{array}{l}\text { Hjortebjerg } \\
\text { et al. (44) }\end{array}$ & CS & 198/99 & 58 & MRI-T2 & WMLs & $\begin{array}{l}\text { IGFBP-3 levels } \\
\text { decreased with } \\
\text { WMLs (Breteler } \\
\text { score 0-2) }\end{array}$ & $P<0.05$ & $\begin{array}{l}\text { age, sex, BMI, diabetes, } \\
\text { triglycerides, fasting insulin, 24-h } \\
\text { AMBP, } 24 \text { h heart rate, use of } \\
\text { statins, antihypertensives }\end{array}$ \\
\hline 19 & $\begin{array}{l}\text { Espeland } \\
\text { et al. (45) }\end{array}$ & $L$ & $319 / 164$ (ILI) & $45-76$ & MRI-T1 T2 & WMHV & $\begin{array}{l}\text { lower WMHV } \\
\text { with lifestyle } \\
\text { intervention } \\
\text { participants }\end{array}$ & $P=0.02$ & $\begin{array}{l}\text { intracranial volume, } \\
\text { age, clinical site }\end{array}$ \\
\hline 20 & $\begin{array}{l}\text { Sudre et al. } \\
(46)\end{array}$ & CS & $469 / 178$ & 71.5 & $\begin{array}{l}\text { MRI-T1 } \\
\text { FLAIR }\end{array}$ & WMHV & $\begin{array}{l}\text { Diabetes } \\
\text { mellitus was not } \\
\text { associated with } \\
\text { higher WMHV }\end{array}$ & NS & NO \\
\hline 21 & $\begin{array}{l}\text { de Havenon } \\
\text { et al. (47) }\end{array}$ & $\mathrm{L}$ & $502 / 502$ & $62.7 \pm 5.7$ & MRI & $\begin{array}{l}\text { progression } \\
\text { of WMHV }\end{array}$ & $\begin{array}{l}\text { A1c was not } \\
\text { associated with } \\
\text { WMH } \\
\text { progression }\end{array}$ & NS & NO \\
\hline
\end{tabular}


TABLE 1 | Continued

\begin{tabular}{|c|c|c|c|c|c|c|c|c|c|}
\hline Number & Study & $\begin{array}{l}\text { Study } \\
\text { Design }\end{array}$ & $\begin{array}{l}\text { Subjects (n) } \\
\text { total/type } 2 \\
\text { DM }\end{array}$ & $\begin{array}{c}\text { Mean age } \\
\text { (years) }\end{array}$ & Imaging & $\begin{array}{l}\text { WMH } \\
\text { assessment } \\
\text { method }\end{array}$ & Results & P-value & Adjustments \\
\hline 23 & $\begin{array}{l}\text { Sundar } \\
\text { et al. (49) }\end{array}$ & CS & 58 & 60 & MRI & $\begin{array}{l}\text { PWWMHs } \\
\text { DWMHs }\end{array}$ & No association & NS & NO \\
\hline 24 & $\begin{array}{l}\text { Rist et al. } \\
\text { (25) }\end{array}$ & L & $345 / 50$ & 74.4 & $\begin{array}{l}\text { MRI-T2 } \\
\text { FLAIR }\end{array}$ & WMHV & No association & NS & $\begin{array}{l}\text { age, sex, parent study, Physical } \\
\text { activity, Smoking, Alcohol } \\
\text { consumption, History of } \\
\text { myocardial infarction, History of } \\
\text { revascularization, History of } \\
\text { cancer,History of hypertension, } \\
\text { Hypertension treatment, History } \\
\text { of high cholesterol, Statin use, } \\
\text { History of TIA, History of angina, } \\
\text { Hormone use, BMl }\end{array}$ \\
\hline 26 & $\begin{array}{l}\text { Power et al. } \\
\text { (51) }\end{array}$ & L & 1,851 & 75.3 & MRI-DTI & FA MD & $\begin{array}{l}\text { elevated } \\
\text { glucose in } \\
\text { midlife with } \\
\text { worse late-life } \\
\text { WM } \\
\text { microstructural } \\
\text { (MD) integrity }\end{array}$ & $P=0.0002$ & $\begin{array}{l}\text { age, gender, education, race/ } \\
\text { center, BMI, the square of BMI, } \\
\text { smoking status, APOE E4, the } \\
\text { other vascular risk factors, } \\
\text { antihypertensive medication use, } \\
\text { antidiabetic medication use, } \\
\text { lipid-lowering medications }\end{array}$ \\
\hline 27 & $\begin{array}{l}\text { Schneider } \\
\text { et al. (52) }\end{array}$ & CS & $1,713 / 1113$ & $\begin{array}{c}75.0(\text { No } \\
\text { diabetes)75.2 } \\
\text { (Prediabetes) } \\
75.4(\text { Diabetes } \\
\text { HbA1c }<7.0 \%) \\
75.1(\mathrm{HbA} 1 \mathrm{c} \\
\geq 7.0 \%)\end{array}$ & $\begin{array}{l}\text { MRI-T2 } \\
\text { FLAIR }\end{array}$ & WMH & $\begin{array}{l}\text { diabetes and } \\
\mathrm{HbA} 1 \mathrm{c} \geq 7.0 \% \\
\text { with increased } \\
\text { WMH }\end{array}$ & $P=0.016$ & $\begin{array}{l}\text { age, sex, race/field center, } \\
\text { education, smoking status, } \\
\text { hypertension, cardiovascular } \\
\text { disease, APOE e4 genotype, } \\
\text { total intracranial volume }\end{array}$ \\
\hline 30 & $\begin{array}{l}\text { Shen et al. } \\
\text { (55) }\end{array}$ & CS & $72 / 36$ & $\begin{array}{l}57.61 \pm 6.21 \\
\quad(\mathrm{~T} 2 \mathrm{DM}) \\
56.19 \pm 6.84 \\
\text { (control) }\end{array}$ & MRI-DIR & WMHs & $\begin{array}{l}\text { More WMHs in } \\
\text { the diabetic } \\
\text { group }\end{array}$ & $p=0.0015$ & NO \\
\hline 31 & $\begin{array}{l}\text { Reitz et al. } \\
\text { (56) }\end{array}$ & CS & $618 / 422$ & 80.0 & MRI & WMHV & $\begin{array}{l}\text { higher levels of } \\
\text { HbA1c were } \\
\text { associated with } \\
\text { increased } \\
\text { WMHV }\end{array}$ & $P<0.05$ & $\begin{array}{l}\text { intracranial volume, age, sex, } \\
\text { education, ethnic group, APOE }\end{array}$ \\
\hline 32 & $\begin{array}{l}\text { Nouwen } \\
\text { et al. (57) }\end{array}$ & CS & $53 / 14$ & $\begin{array}{c}16.1 \pm 1.5 \\
(\mathrm{~T} 2 \mathrm{DM}) \\
14.9 \pm 2.00 \\
\text { (Obese) }\end{array}$ & MRI-TBSS & FA & $\begin{array}{l}\text { FA was } \\
\text { negatively } \\
\text { correlated } \\
\text { HOMA-IR }\end{array}$ & $p<0.0001$ & age, BMI, HbA1c \\
\hline
\end{tabular}


TABLE 1 | Continued

\begin{tabular}{|c|c|c|c|c|c|c|c|c|c|}
\hline Number & Study & $\begin{array}{l}\text { Study } \\
\text { Design }\end{array}$ & $\begin{array}{c}\text { Subjects (n) } \\
\text { total/type } 2 \\
\text { DM }\end{array}$ & $\begin{array}{l}\text { Mean age } \\
\text { (years) }\end{array}$ & Imaging & $\begin{array}{l}\text { WMH } \\
\text { assessment } \\
\text { method }\end{array}$ & Results & P-value & Adjustments \\
\hline & & & & $\begin{array}{l}16.4 \pm 1.7 \\
\text { (Controls) }\end{array}$ & & & & & \\
\hline 33 & $\begin{array}{l}\text { Rofey et al. } \\
\text { (58) }\end{array}$ & CS & $25 / 15$ & $\begin{array}{c}\text { 18(T2DM) } \\
\text { 15.4(Obese) } \\
\text { 14.5(Control) }\end{array}$ & MRI-DTI & FA & $\begin{array}{l}\text { lower FA(right } \\
\text { hemisphere) } \\
\text { with T2DM }\end{array}$ & $p=0.0023$ & NO \\
\hline 34 & $\begin{array}{l}\text { Xiong et al. } \\
(59)\end{array}$ & CS & $58 / 30$ & $\begin{array}{c}60.63 \pm 6.01 \\
(\mathrm{~T} 2 \mathrm{DM}) \\
58.54 \pm 6.22 \\
(\mathrm{HC})\end{array}$ & MRI-DKI & MK & $\begin{array}{l}\text { decreased MK } \\
\text { with T2DM }\end{array}$ & $P<0.05$ & $\mathrm{NO}$ \\
\hline 35 & $\begin{array}{l}\text { Xiong et al. } \\
(60)\end{array}$ & CS & $68 / 42$ & $\begin{array}{c}59.05 \pm 6.22 \\
(\mathrm{DM}-\mathrm{NC}) \\
62.75 \pm 5.93 \\
(\mathrm{DM}-\mathrm{MCl}) \\
59.88 \pm 6.17 \\
\text { (Controls) }\end{array}$ & MRI-TBSS & FA MD & $\begin{array}{l}\text { decreased FA } \\
\text { (CT.R and } \\
\text { CP.R) and } \\
\text { increased MD } \\
\text { (RIC.R and } \\
\text { EC.R) with } \\
\text { T2DM }\end{array}$ & $\begin{array}{l}p=0.003 p=0.002 \\
p=0.021 p=0.009\end{array}$ & NO \\
\hline 36 & $\begin{array}{l}\text { Zhuo et al. } \\
(61)\end{array}$ & CS & $66 / 40$ & $\begin{array}{c}41.7 \pm 9.5 \\
(\mathrm{~T} 2 \mathrm{DM}-\mathrm{NC}) \\
47.3 \pm 8.2 \\
(\mathrm{~T} 2 \mathrm{DM}-\mathrm{C}) \\
42.5 \pm 10.4 \\
(\mathrm{HCs})\end{array}$ & MRI-TBSS & FA MD & $\begin{array}{l}\text { no association } \\
\text { in the FA values } \\
\text { between the } \\
\mathrm{HCs} \text { and T2DM- } \\
\mathrm{NC} \\
\text { decreased FA } \\
\text { values and } \\
\text { increased MD in } \\
\text { the T2DM-C }\end{array}$ & $\begin{array}{l}\mathrm{NS} \\
\mathrm{P}<0.05\end{array}$ & $\mathrm{NO}$ \\
\hline 37 & $\begin{array}{l}\text { Gao et al. } \\
(62)\end{array}$ & CS & $116 / 76$ & $\begin{array}{c}65.97 \pm 7.81 \\
(\mathrm{~T} 2 \mathrm{DM}-\mathrm{aMCl}) \\
65.35 \pm 7.77 \\
(\mathrm{~T} 2 \mathrm{DM}-\mathrm{NC}) \\
66.48 \pm 7.46 \\
(\mathrm{HC})\end{array}$ & MRI-DTI & FA & $\begin{array}{l}\text { no association } \\
\text { in the FA values } \\
\text { between T2DM- } \\
\mathrm{NC} \text { patients and } \\
\mathrm{HC} \\
\text { decreased FA } \\
\text { values in T2DM- } \\
\text { aMCl }\end{array}$ & $\begin{array}{l}\text { NS } \\
p<0.05\end{array}$ & $\begin{array}{l}\text { age, sex, education, BMI, } \\
\text { hypertension, hyperlipidemia, } \\
\text { and cerebrovascular disease }\end{array}$ \\
\hline 38 & $\begin{array}{l}\text { Espeland } \\
\text { et al. (42) }\end{array}$ & $L$ & $319 / 319$ & 69 & MRI & WMHV & $\begin{array}{l}\text { Women had a } \\
\text { greater mean } \\
\text { than men in } \\
\text { summed WMH } \\
\text { volumes }\end{array}$ & $\begin{array}{l}(95 \% \mathrm{Cl}): \\
{[0.00002,2.78]}\end{array}$ & $\begin{array}{l}\text { age, systolic and diastolic blood } \\
\text { pressures }\end{array}$ \\
\hline
\end{tabular}

CS, cross-sectional; DKI, diffusion kurtosis imaging; DM, diabetes mellitus; DTI, diffusion tensor imaging; DM-NC,DM with normal cognition; DM-MCI,DM with mild cognitive impairment; FA, anisotropy fraction; FPG, fasting plasma glucose; HbA1C, glycated hemoglobin; L, longitudinal; LA, leukoaraiosis; MD, mean diffusivity; MRI, magnetic resonance imaging; MTI, magnetization transfer imaging; NS, not significant; WMHs, white matter hyperintensities; NAWM, normal-appearing white matter; FLAIR, Fluid attenuated inversion recovery; WML, white matter lesion; DWMLs, deep white matter lesions; PWWMHs, Periventricular white matter hyperintensities; DWMHs, Deep white matter hyperintensities; PVH, Periventricular hyperintensity;

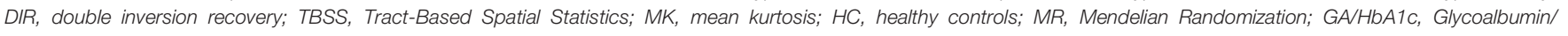
Glycohemoglobin Ratio; IC, intracranial volume.

changes in DTI measurements in T2DM. Persons with T2DM displayed loss of microstructural organization in the association tracts and the forceps minor reflected in a decrease in FA, and not related to age. A study of young and middle-aged adults (40) showed the strongest associations of diabetes and greater fasting blood glucose with reduced FA in WM. Tracts mostly implicated in those associations were the short association fibers, the inferior longitudinal fasciculus, the thalamic radiations, and the corpus callosum. The study found that hyperglycemia is associated with subtle brain injury even in young adults, indicating that brain injury is an early manifestation of impaired glucose metabolism. Two studies of adolescent T2DM and WMLs $(57,58)$ also found a reduction in FA in the T2DM group. Research data suggest that even adolescent T2DM is associated with differences in WM microstructure. A prediabetic study (36) also found WM microstructural damage in prediabetic patients. The predominant DTI abnormalities in prediabetic patients were mainly the reduction of FA values in the right part of the corpus callosum body (bCC), the right superior longitudinal fasciculus (SLF.R), and the left superior longitudinal fasciculus (SLF.L), suggesting that these areas may be the sites of the first lesion. On the other hand, the differences in $\mathrm{MD}, \mathrm{AD}$, and relative anisotropy (RD) between the two groups were not statistically significant in this study, suggesting that FA may be a more sensitive indicator of DTI parameter values. A European Mendelian randomization study (53) also found a significant association between T2DM and FA. Surprisingly, T2DM was not significantly associated with increased MD. It has been suggested that the FA decrease may be modulated more directly by myelin alterations, whereas MD is 
more sensitive to cellularity, edema, and necrosis (62). These results might, therefore, point to demyelination being an important factor in T2DM patients.

Several studies $(32,41,60)$ have also found that T2DM status is simultaneously associated with reduced FA and increased MD. A longitudinal cohort study (51), the ARIC study quantified the relationship between vascular risk factors in midlife and later life and WM microstructural integrity in later life. The results showed that elevated glucose in midlife is related to worse WM microstructural integrity (reduced FA and increased MD) in later life.

In the Maastricht Study, Laura W. Vergoossen et al. (37) used whole-brain white matter tractography to find that prediabetes, T2DM, and continuous measures of hyperglycemia are associated with fewer white matter connections and weaker organization of white matter networks.

However, there are also some studies showing no convincing association between T2DM and poor WM microstructural integrity. Zhuo et al. (61) found that there was no significant difference in the FA values between the healthy controls (HCs) and T2DM patients without peripheral microvascular complications (T2DM-NC groups). Gao also (52) showed the T2DM-NC patients and HC individuals did not reveal any significant differences in WM integrity. However, the integrity of multiple WM tracts was impaired in patients with T2DMaMCI (T2DM with amnesic mild cognitive impairment) compared with HC and T2DM-NC.

The observation of WM microstructures mostly uses DTI technology. DKI was developed to quantify the non-Gaussian property (65). Xiong et al. (59) used DKI to assess WM structure in patients with T2DM. They found T2DM patients had abnormalities, reflected by decreased FA and mean kurtosis $(\mathrm{MK})$, in a variety of white matter regions, as well as in the thalamus and caudate. MK reductions occurred across a more extensive area than DTI metrics, such as in the pontine crossing tract, internal capsule, superior longitudinal fasciculus, corpus callosum, and gray matter structure. It indicated that DKI could provide more changes in the WM microstructure than DTI.

\section{Type 2 Diabetes Mellitus Affects Cognitive Function Through White Matter Hyperintensities}

Although there is evidence that T2DM is independently associated with cognitive decline and $\mathrm{WMH}$ is associated with cognitive deterioration (50), whether T2DM affects cognition through WMH remains controversial. Two prospective cohort studies $(24,41)$ examined the relationship between T2DM and $\mathrm{WMH}$ and cognition at a longitudinal level. Anna Marseglia et al. (24) followed 455 people in the SNAC-K cohort for three or six years and found that T2DM increased the accumulation of WMH. Prediabetes and T2DM were observed to be independently associated with an accelerated decline in cognitive ability. Ben Assayag et al. (50) followed patients with first acute ischemic strokes or transient ischemic attacks (TABASCO cohort) for two years. They found that impaired baseline WM integrity in T2DM was independently associated with worse cognitive test performances two years later. In patients with T2DM, they observed impaired memory, executive function, and attention scores. This could be explained by the sensitivity of executive functioning and processing speed to delicate diffuse vascular-related deterioration in WM integrity. Weinstein's findings (40) suggest that young adults and middle-aged persons with diabetes perform worse on tasks of verbal and visual memory, visual perception and attention, and have more severe white matter microstructural damage (decreased FA) compared to persons without diabetes. Studies of multiethnic groups (34, 35,39 ) have found that T2DM is associated with brain atrophy and WML load, which in turn is associated with poorer cognitive performance. The current analysis of Caucasian and AfricanAmerican groups in the United States with T2DM shows that there is consistent evidence of a correlation between poor performance on global executive function tests and abnormal brain structure. Two studies $(60,62)$ investigated WM microstructural changes by DTI analysis in patients with T2DM with mild cognitive impairment and those with normal cognition. The results showed that the episodic memory and attention function impairment in patients with T2DM has significant correlations with the integrity decline of white matter fibers in the right IFOF and right ILF. Memory changes in patients with T2DM are a gradual, continuous process that may not be adequately reflected by neuropsychological test scores but can be captured in DTI parameters. In a large cohort of older overweight or obese adults with T2DM, differences in brain volumes and white matter disease were apparent between women and men, but these did not account for a lower prevalence of cognitive impairment in women compared with men in this cohort. In the above studies, seven studies $(24,34,35,39,40,50,62)$ adjusted for vascular risk factors such as hypertension, hyperlipidemia cardiovascular, and cerebrovascular diseases. This has implications that the observed relationship is true.

Although most studies have observed pathological vascular changes on MRI in patients with cognitive impairment and T2DM compared to patients without T2DM, other studies (31) have also observed that T2DM with cognitive impairment is mainly characterized by reduced gray matter volume and less disruption of white matter connectivity. These findings suggest that these vascular lesions such as WMH are not important determinants of the severity of cognitive impairment in patients with T2DM, and other etiologies need to be considered.

\section{Relationship Between Type 2 Diabetes Mellitus Microangiopathy, Neuropathy, and White Matter Hyperintensities}

Four studies were found to correlate diabetic microangiopathy, peripheral neuropathy, and WMH $(30,33,43,61)$. Sanahuja et al. (43) observed 312 patients with T2DM. Of these, 153 patients (49\%) had diabetic retinopathy (DR). It was found that the prevalence of $\mathrm{WMH}$ was higher in patients with $\mathrm{DR}$, and the severity of $\mathrm{WMH}$ was associated with the presence of DR. Another study (33) examined 584 patients with T2DM and found that the larger the WMH volume, the higher the UACR and the lower the eGFR. Zhuo et al. (61) found that compared 
with T2DM-NC groups and HCs, the FA values in the corpus callosum, internal capsule, and other regions were significantly decreased in patients with T2DM-C. Sevgi Ferik (30) confirmed in a longitudinal study that there is a statistically significant association between polyneuropathy and Fazekas grade 1 or 2 DWMLs after adjusting for age, duration of T2DM and duration of polyneuropathy symptoms also found to be associated with DWMLs. These studies suggest that the brain may be a target organ for T2DM microangiopathy, similar to other classical target organs, such as the retina or kidney. Interestingly, Mika Sonoda et al. (54) conducted a cross-sectional study of 143 patients with T2DM who were not diagnosed with dementia or stroke. In multiple regression models adjusted for 12 potential confounders, eGFR was not significant in the presence of any type of $\mathrm{WMH}$.

\section{Others}

Four studies examined the association of duration of $\mathrm{DM}(32,33$, 46, 57) and HbAlc values (32, 47, 50, 56) with WMH, respectively. Christiane Reitz et al. (56) used HbAlc as a continuous variable to define the clinical type of dysglycemia. They explored the relationship between clinical types of dysglycemia and brain structure in older persons and found that higher levels of $\mathrm{HbAlc}$ were associated with an increased volume of WMH. The SABRE study (46) showed the duration of diabetes mellitus was only significantly associated with lesion load in the frontal periventricular regions for the European population. Yoshiaki Tamura et al. (50) found that WMH/IC was significantly associated with $\mathrm{GA} / \mathrm{HbA1c}$ in a group of DM patients aged $>65$ years, suggesting glucose variability is the most influential factor, and the avoidance of glucose fluctuations may be a key strategy to prevent WMH. However, in the ACCORD study (47), Adam de Havenon et al. followed up for 40 months and then reported that the strict glycemic control group with $\mathrm{HbAlc}<6.0 \%$ was not associated with $\mathrm{WMH}$ progression. The results of Laura M. Raffield (32) were also not statistically significant, and those of Nouwen et al. (57) were consistent.

Insulin resistance (57), fasting insulin level (48), insulin-like growth factor (IGF) (44), and lifestyle intervention (45) were also found to be significantly associated with WMH. Arie Nouwen et al. (57) reported that an insulin resistance index (HOMA-IR) was the only independent predictor of FA, explaining $8.1 \%$ of the variance in FA in adolescents. Keith A. Hawkins et al. (48) showed that with shared variance controlled for, plasma insulin remained as significant predictors of $\mathrm{WMH}$ volume. The results of Rikke Hjortebjerg (44) showed that IGFBP-3 was negatively correlated with the severity of WMLs. In a longitudinal study (45), participants with T2DM were randomly assigned to receive ten years of lifestyle intervention. It was found that the mean (SE) WMH volume was $28 \%$ lower among lifestyle intervention participants compared with those receiving diabetes support and education. Corneal confocal microscopy (CCM) a surrogate marker of neurodegeneration has shown that the presence of $\mathrm{WMH}$ was associated with corneal nerve loss in patients with acute ischemic stroke after adjusting for age, diabetes, gender, dyslipidemia, and smoking (66).

\section{DISCUSSION}

In this review, we analyzed recently accumulated evidence to explore the link between the presence of T2DM and WMH or its progression over time, and also considered the impact of the relationship between T2DM and WMH on cognitive function. Our study shows that: (a) Both from a macro- and micro-scopic point of view most studies support this relationship of a larger $\mathrm{WMH}$ and a decreased in the integrity of $\mathrm{WMH}$ in T2DM. Using DTI or DKI technology to measure the microstructural changes of WM, most studies have observed a decrease in the integrity of WM in T2DM or prediabetes, especially in the FA values, mainly concentrated in the corpus callosum, combined tract and other parts; (b) From the relationship between brain structural changes and cognition in T2DM, the evidence that poor performance in memory, attention and executive function tests is associated with abnormal brain structure is consistent; (c) Diabetic microangiopathy or peripheral neuropathy may be associated with $\mathrm{WMH}$, suggesting that the brain may be a target organ for T2DM microangiopathy; (d) Laboratory markers such as insulin resistance and fasting insulin levels were significantly associated with WMH, High HbA1c and high glucose variability are associated with WMH but not glycemic control.

The strengths of our study included: (1) We conducted a very thorough and extensive search on PubMed and Cochrane for all types of research in the past five years; (2) We comprehensively explored the relationship between T2DM and $\mathrm{WMH}$ in the elderly, middle-aged and young people, adolescents and different ethnic groups; (3) We analyzed the relationship between T2DM and the macrostructure and microstructural changes of WM from different perspectives.

There were some limitations to this research. (1) The results of this study are limited by the quality of these studies, especially the cross-sectional studies; (2) The heterogeneity of results may be due to differences in blood glucose measurement and the method of using and explaining the MRI; (3) The sample size of the selected studies was somewhat small and some articles did not provide the necessary information, so it is difficult for us to further analyze their heterogeneity; (4) Subgroup analysis was not possible due to the small number of studies; (5) We only included articles published in the English language. In future research, more advanced multiparametric MRI techniques should be used to study the structural changes of WM in T2DM and their effects on cognitive function, as well as potential pathophysiological mechanisms. In addition, future research should also pay more attention to the relationship between prediabetes or T2DM in adolescents, middle-aged and young adults, and abnormal WM structure, because abnormal WM structure may already occur in prediabetes or adolescent type 2 diabetes.

\section{CONCLUSION}

Brain structural changes and associated cognitive decline in patients with T2DM can be detected by neuroimaging. Compared with structural magnetic resonance alone, advanced and novel functional magnetic resonance techniques are expected to reveal subtler brain 
changes. T2DM is directly associated with reduced WM integrity, which, in turn, leads to cognitive decline. Whether cognitive changes can be captured by multiparametric functional magnetic resonance imaging still requires high-quality, longitudinal, and long-term research to improve our understanding of this connection and cognition.

\section{DATA AVAILABILITY STATEMENT}

The original contributions presented in the study are included in the article/supplementary materials; further inquiries can be directed to the corresponding author.

\section{AUTHOR CONTRIBUTIONS}

$\mathrm{W}-\mathrm{DQ}$ and WL conceptualized and designed the research. $\mathrm{W}$-DQ and W-MM acquired the data. X-XS analyzed and

\section{REFERENCES}

1. Hase Y, Horsburgh K, Ihara M, Kalaria RN. White matter degeneration in vascular and other ageing-related dementias. J Neurochem (2018) 144(5):61733. doi: $10.1111 /$ jnc. 14271

2. Wardlaw JM, Smith EE, Biessels GJ, et al. Neuroimaging standards for research into small vessel disease and its contribution to ageing and neurodegeneration. Lancet Neurol (2013) 12(8):822-38. doi: 10.1016/S1474-4422(13)70060-7

3. No H-J, Yi H-A, Won KS, et al. Association between white matter lesions and cerebral glucose metabolism in patients with cognitive impairment. Rev Esp Med Nucl Imagen Mol (English Edition) (2019) 38(3):160-6. doi: 10.1016/ j.remnie.2019.01.005

4. Yi HA, Won KS, Chang HW, et al. Association between white matter lesions and cerebral Abeta burden. PloS One (2018) 13(9):e0204313. doi: 10.1371/ journal.pone.0204313

5. Wagner M, Helfrich M, Volz S, Magerkurth J, Blasel S, Porto L, et al. Quantitative T2, T2*, and T2' MR imaging in patients with ischemic leukoaraiosis might detect microstructural changes and cortical hypoxia. Neuroradiology (2015) 57(10):1023-30. doi: 10.1007/s00234-015-1565-x

6. Moroni F, Ammirati E, Rocca MA, Filippi M, Magnoni M, Camici PG. Cardiovascular disease and brain health: Focus on white matter hyperintensities. Int J Cardiol Heart Vasc (2018) 19:63-9. doi: 10.1016/j.ijcha.2018.04.006

7. Han F, Zhai FF, Wang Q, Zhou LX, Ni J, Yao M, et al. Prevalence and Risk Factors of Cerebral Small Vessel Disease in a Chinese Population-Based Sample. J Stroke (2018) 20(2):239-46. doi: 10.5853/jos.2017.02110

8. Alber J, Alladi S, Bae HJ, Barton DA, Beckett LA, Bell JM, et al. White matter hyperintensities in vascular contributions to cognitive impairment and dementia (VCID): Knowledge gaps and opportunities. Alzheimers Dement (N Y) (2019) 5:107-17. doi: 10.1016/j.trci.2019.02.001

9. Debette S, Markus HS. The clinical importance of white matter hyperintensities on brain magnetic resonance imaging: systematic review and meta-analysis. BMJ (2010) 341:c3666. doi: 10.1136/bmj.c3666

10. Habes M, Erus G, Toledo JB, Zhang T, Bryan N, Launer LJ, et al. White matter hyperintensities and imaging patterns of brain ageing in the general population. Brain (2016) 139(Pt 4):1164-79. doi: 10.1093/brain/aww008

11. Gottesman RF, Coresh J, Catellier DJ, et al. Blood pressure and white-matter disease progression in a biethnic cohort: Atherosclerosis Risk in Communities (ARIC) study. Stroke (2010) 41(1):3-8. doi: 10.1161/STROKEAHA. 109.566992

12. Godin O, Tzourio C, Maillard P, Mazoyer B, Dufouil C. Antihypertensive treatment and change in blood pressure are associated with the progression of white matter lesion volumes: the Three-City (3C)-Dijon Magnetic Resonance interpreted the data. T-XL performed the statistical analysis. W-DQ and C-HX wrote the manuscript. WL and LX critically revised the manuscript for intellectual content. All authors contributed to the article and approved the submitted version.

\section{FUNDING}

Funding of the study was provided by the National Key Research and development Plan "New Strategy on Pathogenesis and Clinical Diagnosis and Treatment of Chronic Cerebral Small Vessels" (No. 2016YFC1300600).

\section{ACKNOWLEDGMENTS}

We would like to acknowledge the hard and dedicated work of all the staff that implemented the intervention and evaluation components of the study.

Imaging Study. Circulation (2011) 123(3):266-73. doi: 10.1161/ CIRCULATIONAHA.110.961052

13. Zheng Y, Ley SH, Hu FB. Global aetiology and epidemiology of type 2 diabetes mellitus and its complications. Nat Rev Endocrinol (2018) 14(2):88-98. doi: 10.1038/nrendo.2017.151

14. Kyrou I, Tsigos C, Mavrogianni C, Cardon G, Van Stappen V, Latomme J, et al. Sociodemographic and lifestyle-related risk factors for identifying vulnerable groups for type 2 diabetes: a narrative review with emphasis on data from Europe. BMC Endocr Disord (2020) 20(Suppl 1):134. doi: 10.1186/ s12902-019-0463-3

15. Cho NH, Shaw JE, Karuranga S, Huang Y, da Rocha Fernandes JD, Ohlrogge AW, et al. IDF Diabetes Atlas: Global estimates of diabetes prevalence for 2017 and projections for 2045. Diabetes Res Clin Pract (2018) 138:271-81. doi: 10.1016/j.diabres.2018.02.023

16. Jia W, Xu A, Chen A, Wu J, Ye J. Chronic vascular complications in diabetes. J Diabetes Res (2013) 2013:858746. doi: 10.1155/2013/858746

17. Emerging Risk Factors Collaboration, Sarwar N, Gao P, Seshasai SR, Gobin R, Kaptoge S, et al. Diabetes mellitus, fasting blood glucose concentration, and risk of vascular disease: a collaborative meta-analysis of 102 prospective studies. Lancet (2010) 375(9733):2215-22. doi: 10.1016/S0140-6736(10)60484-9

18. Munoz-Rivas N, Mendez-Bailon M, Hernandez-Barrera V, et al. Type 2 Diabetes and Hemorrhagic Stroke: A Population-Based Study in Spain from 2003 to 2012. J Stroke Cerebrovasc Dis (2016) 25(6):1431-43. doi: 10.1016/ j.jstrokecerebrovasdis.2016.02.031

19. Pal K, Mukadam N, Petersen I, et al. Mild cognitive impairment and progression to dementia in people with diabetes, prediabetes and metabolic syndrome: a systematic review and meta-analysis. Soc Psychiatry Psychiatr Epidemiol (2018) 53(11):1149-60. doi: 10.1007/s00127-018-1581-3

20. Dybjer E, Nilsson PM, Engström G, Helmer C, Nägga K. Pre-diabetes and diabetes are independently associated with adverse cognitive test results: a cross-sectional, population-based study. BMC Endocr Disord (2018) 18(1):91. doi: 10.1186/s12902-018-0318-3

21. Swardfager W, Macintosh BJ. Depression, Type 2 Diabetes, and Poststroke Cognitive Impairment. Neurorehabil Neural Repair (2017) 31(1):48-55. doi: $10.1177 / 1545968316656054$

22. de Bresser J, Tiehuis AM, van den Berg E, Reijmer YD, Jongen C, Kappelle LJ, et al. Progression of cerebral atrophy and white matter hyperintensities in patients with type 2 diabetes. Diabetes Care (2010) 33(6):1309-14. doi: $10.2337 / \mathrm{dc} 09-1923$

23. Mankovsky B, Zherdova N, Van Den Berg E, et al. Cognitive functioning and structural brain abnormalities in people with Type 2 diabetes mellitus. Diabetes Med (2018) 35(12):1663-70. doi: 10.1111/dme.13800 
24. Marseglia A, Fratiglioni L, Kalpouzos G, Wang R, Bäckman L, Xu W. Prediabetes and diabetes accelerate cognitive decline and predict microvascular lesions: A population-based cohort study. Alzheimers Dement (2019) 15(1):25-33. doi: 10.1016/j.jalz.2018.06.3060

25. Rist PM, Buring JE, Rexrode KM, et al. Prospectively collected lifestyle and health information as risk factors for white matter hyperintensity volume in stroke patients. Eur J Epidemiol (2019) 34(10):957-65. doi: 10.1007/s10654-019-00546-x

26. Del Bene A, Ciolli L, Borgheresi L, et al. Is type 2 diabetes related to leukoaraiosis? an updated review. Acta Neurol Scand (2015) 132(3):147-55. doi: 10.1111/ane.12398

27. De Bresser J, Kuijf HJ, Zaanen K, et al. White matter hyperintensity shape and location feature analysis on brain MRI; proof of principle study in patients with diabetes. Sci Rep (2018) 8(1):1893. doi: 10.1038/s41598-018-20084-y

28. Lucatelli P, Montisci R, Sanfilippo R, et al. Is there an association between leukoaraiosis volume and diabetes? J Neuroradiol (2016) 43(4):273-9. doi: 10.1016/j.neurad.2015.11.003

29. de Groot M, Ikram MA, Akoudad S, Krestin GP, Hofman A, van der Lugt A, et al. Tract-specific white matter degeneration in aging: The Rotterdam Study. Alzheimers Dement (2015) 11(3):321-30. doi: 10.1016/j.jalz.2014.06.011

30. Ferik S, Güven H, Ateş MP, et al. Diabetic polyneuropathy, deep white matter lesions, and carotid atherosclerosis: is there any association? Neurol Sci (2017) 39(1):103-10. doi: 10.1007/s10072-017-3160-x

31. Groeneveld O, Reijmer Y, Heinen R, et al. Brain imaging correlates of mild cognitive impairment and early dementia in patients with type 2 diabetes mellitus. Nutr Metab Cardiovasc Dis (2018) 28(12):1253-60. doi: 10.1016/ j.numecd.2018.07.008

32. Raffield LM, Cox AJ, Freedman BII, et al. Analysis of the relationships between type 2 diabetes status, glycemic control, and neuroimaging measures in the Diabetes Heart Study Mind. Acta Diabetol (2016) 53(3):439-47. doi: 10.1007/ s00592-015-0815-z

33. Freedman BII, Sink KM, Hugenschmidt CE, et al. Associations of Early Kidney Disease With Brain Magnetic Resonance Imaging and Cognitive Function in African Americans With Type 2 Diabetes Mellitus. Am J Kidney Dis (2017) 70(5):627-37. doi: 10.1053/j.ajkd.2017.05.006

34. Hughes TM, Sink KM, Williamson JD, et al. Relationships between cerebral structure and cognitive function in African Americans with type 2 diabetes. J Diabetes its Complications (2018) 32(10):916-21. doi: 10.1016/j.jdiacomp.2018.05.017

35. Whitlow CT, Sink KM, Divers J, et al. Effects of Type 2 Diabetes on Brain Structure and Cognitive Function: African American-Diabetes Heart Study MIND. AJNR Am J Neuroradiol (2015) 36(9):1648-53. doi: 10.3174/ajnr.A4321

36. Liang M, Cai X, Tang Y, Yang XL, Fang J, Li J, et al. Diffusion tensor imaging of white matter in patients with prediabetes by trace-based spatial statistics. J Magn Reson Imaging (2019) 49(4):1105-12. doi: 10.1002/jmri.26290

37. Vergoossen LW, Schram MT, de Jong JJ, Stehouwer CD, Schaper NC, Henry RM, et al. White Matter Connectivity Abnormalities in Prediabetes and Type 2 Diabetes: The Maastricht Study. Diabetes Care (2020) 43(1):201-8. doi: $10.2337 / \mathrm{dc} 19-0762$

38. Van Agtmaal MJM, Houben A, De Wit V, et al. Prediabetes Is Associated With Structural Brain Abnormalities: The Maastricht Study. Diabetes Care (2018) 41(12):2535-43. doi: 10.2337/dc18-1132

39. Hsu FC, Sink KM, Hugenschmidt CE, et al. Cerebral Structure and Cognitive Performance in African Americans and European Americans With Type 2 Diabetes. J Gerontol A Biol Sci Med Sci (2018) 73(3):407-14. doi: 10.1093/ gerona/glx 255

40. Galit Weinstein P, Pauline Maillard P, Jayandra J, Himali P, et al. Glucose indices are associated with cognitive and structural brain measures in young adults. Am Acad Neurol (2015) 84:1-9. doi: 10.1212/WNL.0000000000001655

41. Ben Assayag E, Eldor R, Korczyn AD, Kliper E, Shenhar-Tsarfaty S, Tene O, et al. Type 2 Diabetes Mellitus and Impaired Renal Function Are Associated With Brain Alterations and Poststroke Cognitive Decline. Stroke (2017) 48 (9):2368-74. doi: 10.1161/STROKEAHA.117.017709

42. Lin Q, Huang WQ, Ma QL, Lu CX, Tong SJ, Ye JH, et al. Incidence and risk factors of leukoaraiosis from 4683 hospitalized patients. Medicine (2017) 96 (39):e7682. doi: 10.1097/MD.0000000000007682

43. Sanahuja J, Alonso N, Diez J, Ortega E, Rubinat E, Traveset A, et al. Increased Burden of Cerebral Small Vessel Disease in Patients With Type 2 Diabetes and Retinopathy. Diabetes Care (2016) 39(9):1614-20. doi: 10.2337/dc15-2671
44. Hjortebjerg R, Laugesen E, Hoyem P, et al. The IGF system in patients with type 2 diabetes: associations with markers of cardiovascular target organ damage. Eur J Endocrinol (2017) 176(5):521-31. doi: 10.1530/EJE-16-0940

45. Espeland MA, Erickson K, Neiberg RH, Jakicic JM, Wadden TA, Wing RR, et al. Brain and White Matter Hyperintensity Volumes After 10 Years of Random Assignment to Lifestyle Intervention. Diabetes Care (2016) 39:76471. doi: $10.2337 / \mathrm{dc} 15-2230$

46. Sudre CH, Smith L, Atkinson D, Chaturvedi N, Ourselin S, Barkhof F, et al. Cardiovascular Risk Factors and White Matter Hyperintensities: Difference in Susceptibility in South Asians Compared With Europeans. J Am Heart Assoc (2018) 7(21):e010533. doi: 10.1161/JAHA.118.010533

47. de Havenon A, Majersik JJ, Tirschwell DL, McNally JS, Stoddard G, Rost NS. Blood pressure, glycemic control, and white matter hyperintensity progression in type 2 diabetics. Am Acad Neurol (2019) 92(11):e1168-75. doi: 10.1212/ WNL.0000000000007093

48. Hawkins KA, Emadi N, Pearlson GD, Winkler AM, Taylor B, Dulipsingh L, et al. Hyperinsulinemia and elevated systolic blood pressure independently predict white matter hyperintensities with associated cognitive decrement in the middle-aged offspring of dementia patients. Metab Brain Dis (2017) 32 (3):849-57. doi: 10.1007/s11011-017-9980-9

49. Sundar U, Manwatkar AA, Joshi AR, Bhandarkar P. The Effect of Hypertension and Diabetes Mellitus on White Matter Changes in MRI Brain: A Comparative Study between Patients with Alzheimer's Disease and an Age-matched Control Group. J Assoc Physicians India (2019) 67(4):14-7.

50. Tamura Y, Kimbara Y, Yamaoka T, Sato K, Tsuboi Y, Kodera R, et al. White Matter Hyperintensity in Elderly Patients with Diabetes Mellitus Is Associated with Cognitive Impairment, Functional Disability, and a High Glycoalbumin/ Glycohemoglobin Ratio. Front Aging Neurosci (2017) 9:220. doi: 10.3389/ fnagi.2017.00220

51. Power MC, Tingle JV, Reid RI, Huang J, Sharrett AR, Coresh J, et al. Midlife and Late-Life Vascular Risk Factors and White Matter Microstructural Integrity: The Atherosclerosis Risk in Communities Neurocognitive Study. $J$ Am Heart Assoc (2017) 6(5):e005608. doi: 10.1161/JAHA.117.005608

52. Schneider ALC, Selvin E, Sharrett AR, Griswold M, Coresh J, Jack CR Jr, et al. Diabetes, Prediabetes, and Brain Volumes and Subclinical Cerebrovascular Disease on MRI: The Atherosclerosis Risk in Communities Neurocognitive Study (ARIC-NCS). Diabetes Care (2017) 40(11):1514-21. doi: 10.2337/dc171185

53. Liu J, Rutten-Jacobs L, Liu M, Markus HS, Traylor M. Causal Impact of Type 2 Diabetes Mellitus on Cerebral Small Vessel Disease: A Mendelian Randomization Analysis. Stroke (2018) 49(6):1325-31. doi: 10.1161/ STROKEAHA.117.020536

54. Sonoda M, Shoji T, Kuwamura Y, Okute Y, Naganuma T, Shima H, et al. Plasma homocysteine and cerebral small vessel disease as possible mediators between kidney and cognitive functions in patients with diabetes mellitus. $S c i$ Rep (2017) 7(1):4382. doi: 10.1038/s41598-017-04515-w

55. Shen Y, Zhao B, Yan L, Jann K, Wang G, Wang J, et al. Cerebral Hemodynamic and White Matter Changes of Type 2 Diabetes Revealed by Multi-TI Arterial Spin Labeling and Double Inversion Recovery Sequence. Front Neurol (2017) 8:717. doi: 10.3389/fneur.2017.00717

56. Reitz C, Guzman VA, Narkhede A, DeCarli C, Brickman AM, Luchsinger JA. Relation of Dysglycemia to Structural Brain Changes in a Multiethnic Elderly Cohort. J Am Geriatr Soc (2017) 65(2):277-85. doi: 10.1111/jgs.14551

57. Nouwen A, Chambers A, Chechlacz M, et al. Microstructural abnormalities in white and gray matter in obese adolescents with and without type 2 diabetes. NeuroImage Clin (2017) 16:43-51. doi: 10.1016/j.nicl.2017.07.004

58. Rofey DL, Arslanian SA, El Nokali NE, et al. Brain volume and white matter in youth with type 2 diabetes compared to obese and normal weight, nondiabetic peers: A pilot study. Int J Dev Neurosci (2015) 46:88-91. doi: 10.1016/ j.ijdevneu.2015.07.003

59. Xiong Y, Sui $\mathrm{Y}$, Zhang $\mathrm{S}$, et al. Brain microstructural alterations in type 2 diabetes: diffusion kurtosis imaging provides added value to diffusion tensor imaging. Eur Radiol (2018) 29(4):1997-2008. doi: 10.1007/s00330-018-5746-y

60. Xiong Y, Sui Y, Xu Z, Zhang Q, Karaman MM, Cai K, et al. A Diffusion Tensor Imaging Study on White Matter Abnormalities in Patients with Type 2 Diabetes Using Tract-Based Spatial Statistics. AJNR Am J Neuroradiol (2016) 37(8):1462-9. doi: 10.3174/ajnr.A4740 
61. Zhuo Y, Fang F, Lu L, Li T, Lian J, Xiong Y, et al. White matter impairment in type 2 diabetes mellitus with and without microvascular disease. NeuroImage Clin (2019) 24:101945. doi: 10.1016/j.nicl.2019.101945

62. Gao S, Chen Y, Sang F, Yang Y, Xia J, Li X, et al. White matter microstructural change contributes to worse cognitive function in type 2 diabetes mellitus patients. Diabetes Care (2019) 68(11):2085-94. doi: 10.2337/db19-0233

63. van Bussel FCG, Backes WH, Hofman PAM, van Oostenbrugge RJ, van Boxtel MPJ, Verhey FRJ, et al. Cerebral Pathology and Cognition in Diabetes: The Merits of Multiparametric Neuroimaging. Front Neurosci (2017) 11:188. doi: 10.3389/fnins.2017.00188

64. Tournier JD, Mori S, Leemans A. Diffusion tensor imaging and beyond. Magn Reson Med (2011) 65(6):1532-56. doi: 10.1002/mrm.22924

65. Hui ES, Cheung MM, Qi L, et al. Towards better MR characterization of neural tissues using directional diffusion kurtosis analysis. Neuroimage (2008) 42(1):122-34. doi: 10.1016/j.neuroimage.2008.04.237
66. Kamran S, Khan A, Salam A, et al. Cornea: A Window to White Matter Changes in Stroke; Corneal Confocal Microscopy a Surrogate Marker for the Presence and Severity of White Matter Hyperintensities in Ischemic Stroke. J Stroke Cerebrovasc Dis (2020) 29(3):104543. doi: 10.1016/j.jstrokecerebrovasdis.2019.104543

Conflict of Interest: The authors declare that the research was conducted in the absence of any commercial or financial relationships that could be construed as a potential conflict of interest.

Copyright (c) 2020 Wang, Wang, Wei, Xia, Tian, Cui and Li. This is an open-access article distributed under the terms of the Creative Commons Attribution License (CC BY). The use, distribution or reproduction in other forums is permitted, provided the original author(s) and the copyright owner(s) are credited and that the original publication in this journal is cited, in accordance with accepted academic practice. No use, distribution or reproduction is permitted which does not comply with these terms. 